\title{
Oocyte-expressed yes-associated protein is a key activator of the early zygotic genome in mouse
}

\author{
Chao $\mathrm{Yu}^{1,{ }^{*}}$, Shu-Yan $\mathrm{Ji}^{1,{ }^{*}}$, Yu-Jiao Dang ${ }^{2,}$, , Qian-Qian Sha ${ }^{1}$, Yi-Feng Yuan ${ }^{3}$, Jian-Jie Zhou ${ }^{1}$, Li-Ying Yan ${ }^{3}$, \\ Jie Qiao ${ }^{3}$, Fuchou Tang ${ }^{2}$, Heng-Yu Fan ${ }^{1}$ \\ ${ }^{1}$ Life Sciences Institute, Zhejiang University, Hangzhou 310058, China; ${ }^{2}$ Biodynamic Optical Imaging Center, College of Life Sci- \\ ences, Peking University, Beijing 100871, China; ${ }^{3}$ Center for Reproductive Medicine, Department of Obstetrics and Gynecology, \\ Peking University Third Hospital, Beijing 100191, China
}

In early mammalian embryos, the genome is transcriptionally quiescent until the zygotic genome activation (ZGA) which occurs 2-3 days after fertilization. Despite a long-standing effort, maternal transcription factors regulating this crucial developmental event remain largely elusive. Here, using maternal and paternal mouse models of Yap1 deletion, we show that maternally accumulated yes-associated protein (YAP) in oocyte is essential for ZGA. Maternal Yap1-knockout embryos exhibit a prolonged two-cell stage and develop into the four-cell stage at a much slower pace than the wild-type controls. Transcriptome analyses identify YAP target genes in early blastomeres; two of which, $R p l 13$ and $R r m 2$, are required to mediate maternal YAP's effect in conferring developmental competence on preimplantation embryos. Furthermore, the physiological YAP activator, lysophosphatidic acid, can substantially improve early development of wild-type, but not maternal Yap1-knockout embryos in both oviduct and culture. These observations provide insights into the mechanisms of ZGA, and suggest potentials of YAP activators in improving the developmental competence of cultured embryos in assisted human reproduction and animal biotechnology.

Keywords: embryo; oocyte; YAP; developmental competence; zygotic genome activation

Cell Research (2016) 26:275-287. doi:10.1038/cr.2016.20; published online 23 February 2016

\section{Introduction}

Mammalian oocytes, upon fertilization, are reprogrammed from a highly specialized, non-proliferative, meiotic state to totipotent, mitotic embryos, which subsequently gain the ability to proliferate, differentiate and develop into new individuals $[1,2]$. A critical step in early development is the maternal-zygotic transition (MZT) occurring 2-3 days after fertilization in mice, which is controlled by maternally deposited RNAs and proteins $[3,4]$. Initially, the zygotic genome is transcriptionally quiescent; however, after MZT, the zygotic genome becomes active and takes control of development [5, $6]$. There is a long-standing question as which maternal

*These three authors contributed equally to this work.

Correspondence: Heng-Yu Fan

Tel: +86-571-88981370

E-mail: hyfan@zju.edu.cn

Received 27 October 2015; revised 15 December 2015; accepted 17 December 2015; published online 23 February 2016 factors regulate MZT, and, particularly, which maternal transcription factor(s) regulate zygotic genome activation (ZGA).

Clinically, many in vitro matured human oocytes exhibit developmental arrest at different stages after fertilization, owing to genetic variations or in some cases, inappropriate culture conditions [7-9]. Therefore, understanding the maternal factors and molecular mechanisms underpinning the developmental competence of preimplantation embryos is significant to the improvement of the success rate in assisted reproductive technology.

The zinc-finger protein Zelda is a key activator of the early zygotic genome in Drosophila [10]. However, the gene encoding this protein $(Z l d)$ is not conserved in mammals, indicating that different genes or mechanisms may be involved. Transcriptome analyses has revealed that Yap 1, which encodes the yes-associated protein (YAP), is highly expressed in human and mouse oocytes and early embryos $[11,12]$. YAP is a transcriptional co-activator of TEAD family transcriptional factors (TEAD1-4) [13, 14]. Zygotically expressed TEAD4 is 
required for the specification of trophectoderm (TE) and inner cell mass (ICM) in blastocysts by inducing the expression of CDX2 in TE cells $[15,16]$. However, the maternal functions of YAP and TEADs in oocyte development and MZT have not been investigated.

In this study, we used maternal and paternal mouse models of Yapl deletion to elucidate the function of maternal YAP in ZGA. By targeting the expression of key early zygotic genes, maternal YAP renders preimplantation embryos developmentally competent. Furthermore, the physiological YAP activator, lysophosphatidic acid (LPA), stimulates early embryonic development. These observations provide insights into the mechanisms of ZGA in mammals, and offer potential new approaches in assisted reproductive technology.

\section{Results}

Yap1 is highly expressed in mammalian oocytes and early embryos

By comparing the transcriptomes of human/mouse oocytes and somatic cells, we found that the transcription coactivator Yapl is predominantly expressed in oocytes and preimplantation embryos, but the other TEAD coactivator Taz is not (Figure 1A and 1B). This observation suggests that YAP may have an important role in ZGA.

Immunofluorescent staining revealed that YAP is expressed in mouse oocytes and early embryos; however, the subcellular distribution varies (Figure 1C and 1D). YAP is evenly distributed in oocytes at germinal vesicle (GV) stage, but is gradually translocated from cytoplasm to nucleus after fertilization. At the morula and blastocyst stages, the nuclei of outer layer blastomeres accumulate significantly higher levels of YAP than inner layer blastomeres (Figure 1C and 1D).

Contributions of maternal and paternal Yapl expression to early embryonic development

To investigate the function of YAP in mouse oocytes, we selectively deleted Yap1 in oocytes by crossing Yapl $^{f l f l}$ mice with transgenic Gdf9-Cre mice [17, 18]. YAP expression in oocytes is abolished in $\operatorname{Yap}^{f l f t} ; G d f 9_{-}$ Cre females (Figure 1E-1G). We then studied the development of zygotes derived from Yapl-knockout oocytes. When fertilized by wild-type (WT) spermatozoa, the resulting maternal Yap1-knockout embryos $\left(\right.$ Yapl $\left.^{\text {P-1/3+}}\right)$ had a prolonged two-cell stage and developed into the fourcell stage at a much slower rate when compared with that of WT controls (Yapl $1^{\text {++/ }}$; Figure 2A-2C). About twothirds $(63.4 \%)$ of the Yap1 $1^{\text {क-1/+ }}$ embryos died before compaction. Only $28.8 \%$ of $\mathrm{Yapl}^{\text {P-/ }} \bar{\delta}^{+}$embryos developed to the blastocyst stage (Figure 2D).
We further analyzed the fertility of Yap $^{f l f f l}$; Gdf9Cre females by crossing them to adult WT males. In a 6-month fertility test, Yap $1^{f l / f l}$;Gdf9-Cre females were subfertile and produced only $2.74 \pm 0.24$ pups per litter, whereas WT littermates produced $7.97 \pm 0.37$ pups

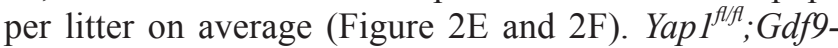
Cre females also exhibited a progressive loss in fertility - they gave birth to 3-5 pups in the first 1-2 litters but gradually produced fewer pups or even became infertile (Figure 2F).

Because the paternal Yapl allele is intact in Yap1 $1^{\text {P-13+ }}$ embryos, we analyzed the expression of YAP in these embryos (Figure 2G-2J). YAP is weakly expressed in Yap $^{\$-1{ }^{+}+}$embryos at four- and eight-cell stages (Figure 2I and 2J). Notably, YAP expression was restored in blastocysts developed from maternal Yap1-deleted zygotes (Figure $2 \mathrm{G}$ ). These results suggest that the expression of paternal Yap1 rescues the developmental defects in some embryos (Figure 2A). To test this hypothesis, we developed Yap $1^{f /-}$;Ddx4-Cre mice, with Yap1 knockout in the male germline. Yapl ${ }^{f / /} ; D d x 4$-Cre males were fertile and had normal spermatogenesis (Supplementary information, Figure S1). Yap1 $1^{\nexists / f}$; Gdf9-Cre females were infertile when mated to these males. YAP expression was completely ablated in Yap1 $1^{\text {P- }}$ - embryos (Figure $2 \mathrm{~A}$ and $2 \mathrm{H}-$ 2J), which exhibit severe developmental defects (Figure 2C). Specifically, Yap1 $1^{\text {क- } / \hat{\delta}^{-}}$embryos had significant growth retardation at two to four-cell stages, and their development was blocked completely before compaction (Figure 2C and 2D).

Owing to the known functions of YAP in cell proliferation, apoptosis and tumorigenesis, YAP may play a role in normal ovarian development and function [1922]. Surprisingly, oocyte-specific deletion of YAP did not affect primordial follicle formation, activation and folliculogenesis. Yap ${ }^{f l / f l}$; Gdf9-Cre females ovulated normal MII oocytes with well-organized spindles (Supplementary information, Figure S2). Moreover, the ovaries of Yap $^{f l f l}$; Gdf9-Cre females contained similar number of developing follicles as those of WT females. Aged (7 months old) Yap $1^{f / f} ; G d f 9-C r e$ females did not show signs of premature ovarian failure (Supplementary information, Figure S2B). These results indicate that, despite its important functions in somatic cells, YAP is dispensable for the survival, growth and maturation of mouse oocytes.

Fertilization and pronucleus formation were not affected by maternal YAP deletion (Figure $3 \mathrm{~A}$ and $3 \mathrm{~B}$ ). Phosphorylated RNA polymerase II at serine-2 (pS2, a marker of RNA polymerase II activation, Figure 3C) and trimethylated histone $\mathrm{H} 3$ at lysine- 4 ( $\mathrm{H} 3 \mathrm{~K} 4 \mathrm{me} 3$, a histone modification that facilitates transcription, Figure 

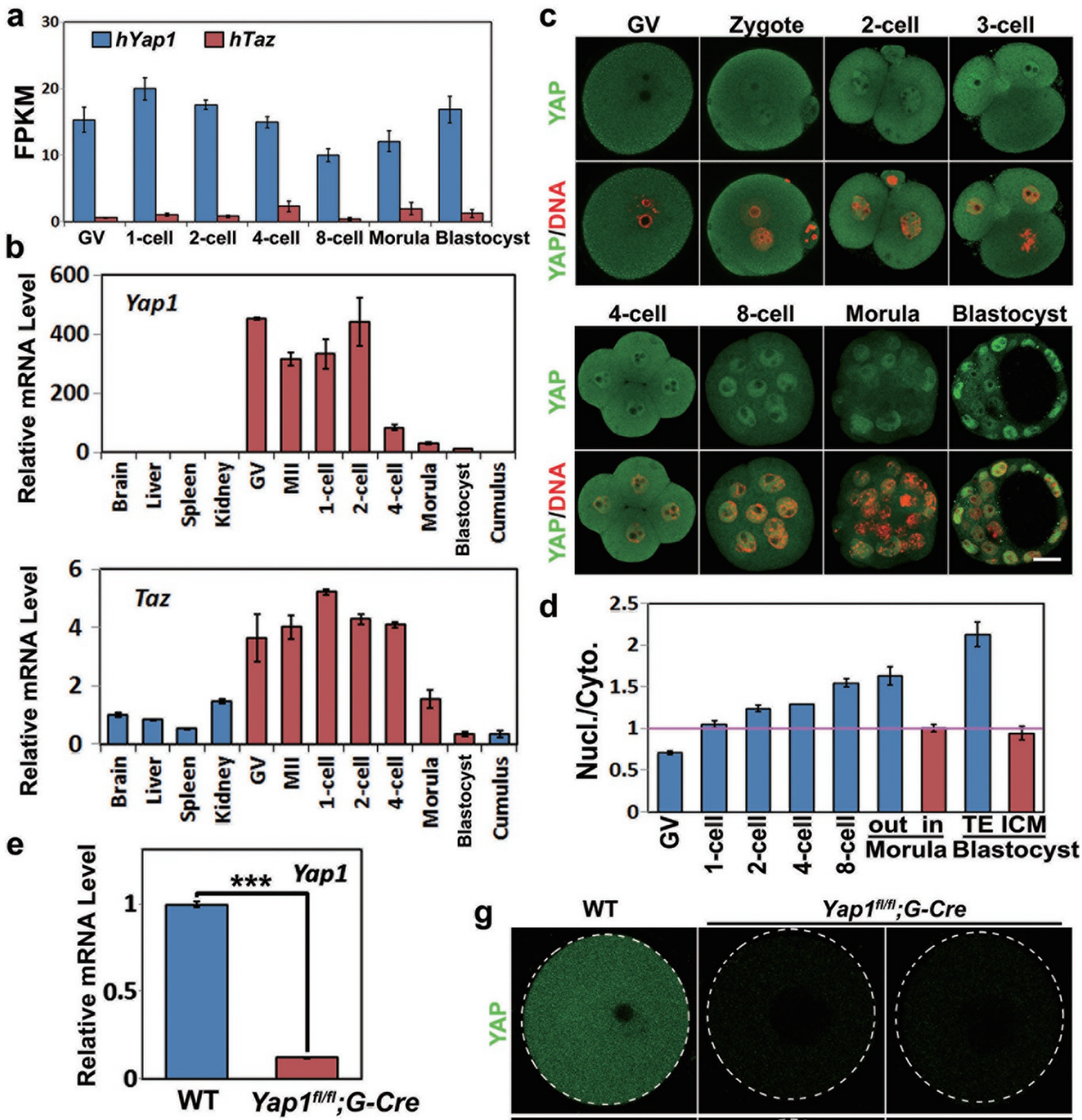

f
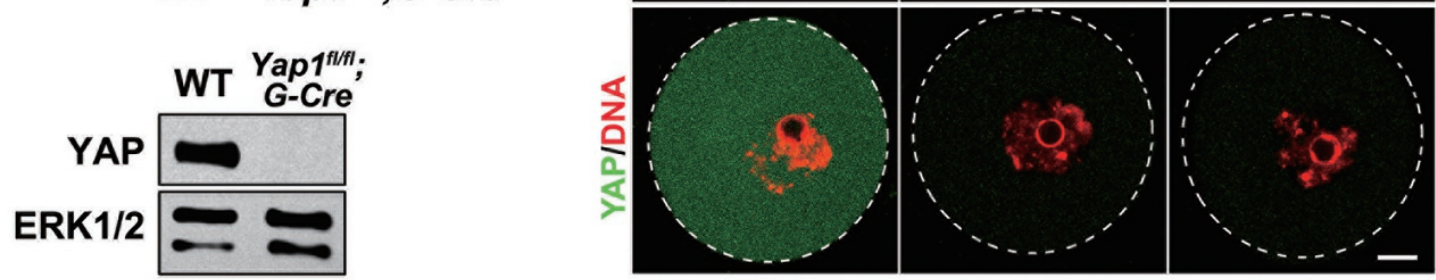

Figure 1 Expression of YAP in preimplantation human and mouse embryos. (A) RNA-seq results showing mRNA levels of Yap1 and Taz in human oocytes and early embryos. FPKM, fragments per kilobase of exon per million fragments mapped. FPKM numbers are extracted from previously published data [11]. Error bars indicate SEM. (B) qRT-PCR results showing mRNA levels of Yap1 and Taz in mouse tissues and oocytes/embryos. Error bars indicate SEM. (C) Immunofluorescent staining of YAP in mouse oocytes and preimplantation embryos. Scale bar, $10 \mu \mathrm{m}$. (D) Ratios of YAP signals in the nucleus (Nucl.) vs cytoplasm (Cyto.) showing the activation of YAP in mouse oocytes and early embryos. Error bars indicate SEM. $n>10$ for oocytes/embryos at each stage. (E-G) qRT-PCR (E), western blot (F) and immunofluorescence (G) results showing deletion of YAP in GV oocytes. G-Cre, Gdf9-Cre. Error bars indicate SEM. ${ }^{* \star \star} P<0.001$, Student's $t$-test. Dashed circles outline the oocytes. Scale bar, $10 \mu \mathrm{m}$. 
a

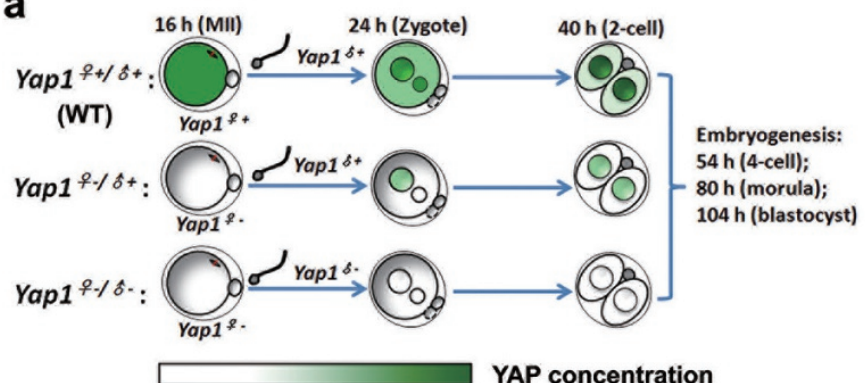

C

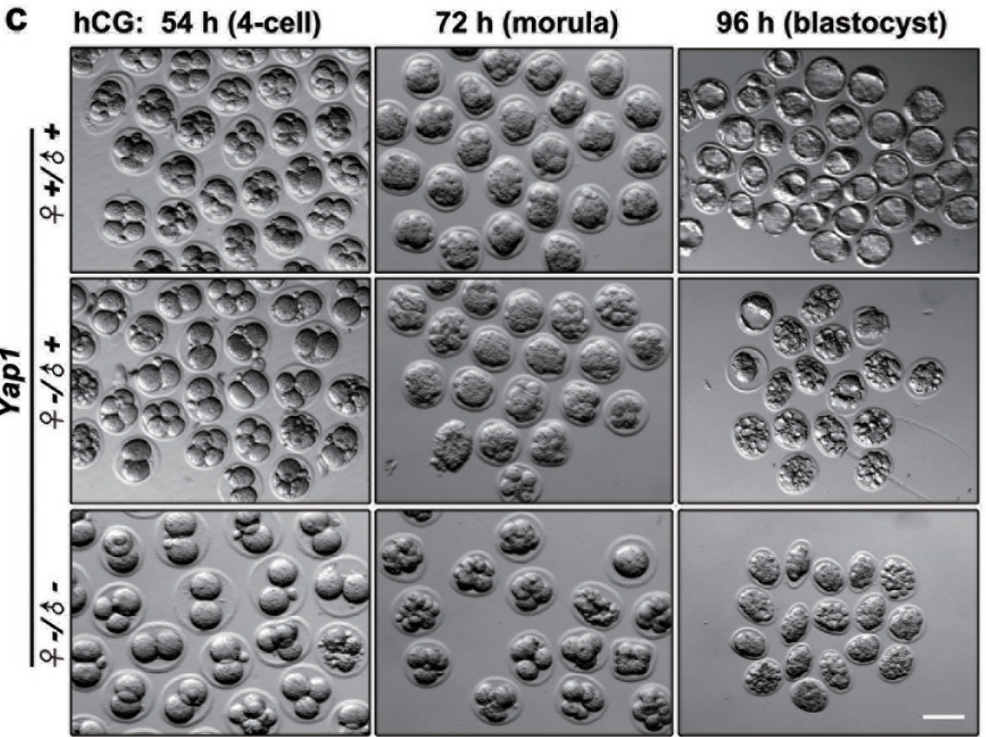

e 10
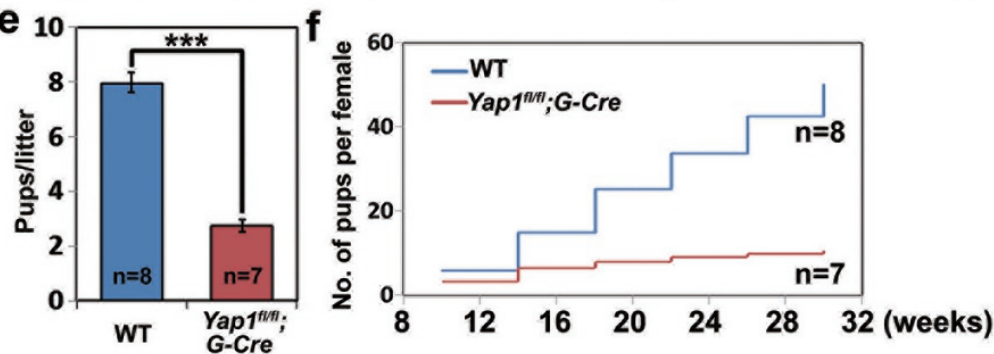

\begin{tabular}{cccccc}
\hline Genotype & $\begin{array}{c}\text { Hours } \\
\text { Post-hCG }\end{array}$ & $\begin{array}{c}\text { 1-cell } \\
(\%)\end{array}$ & $\begin{array}{c}\text { 2-cell } \\
(\%)\end{array}$ & $\begin{array}{c}\text { 3-cell } \\
(\%)\end{array}$ & $\begin{array}{c}\text { 4-cell } \\
(\%)\end{array}$ \\
\hline \multirow{2}{*}{$\begin{array}{c}\text { WT } \\
(n=210)\end{array}$} & $24 \mathrm{~h}$ & 91.6 & 0 & 0 & 0 \\
\cline { 2 - 6 } & $40 \mathrm{~h}$ & 6.3 & 93.7 & 0 & 0 \\
\cline { 2 - 6 } & $54 \mathrm{~h}$ & 0 & 17.1 & 13.3 & 69.5 \\
\hline \multirow{2}{*}{$\begin{array}{c}\text { ㅇ-/ } \hat{y}+ \\
(\mathrm{n}=235)\end{array}$} & $24 \mathrm{~h}$ & 84.8 & 0 & 0 & 0 \\
\cline { 2 - 6 } & $40 \mathrm{~h}$ & 41.3 & 58.7 & 0 & 0 \\
\cline { 2 - 6 } & $54 \mathrm{~h}$ & 4.9 & 63.1 & 10.9 & 20.8 \\
\hline
\end{tabular}

d

\begin{tabular}{|c|c|}
\hline Genotype & $\begin{array}{c}\text { Blastocyst } \\
(\%)\end{array}$ \\
\hline$\underset{(n=92)}{W T}$ & 90.2 \\
\hline $\begin{array}{c}\text { 우 }-/ \hat{o}+ \\
(n=81)\end{array}$ & 28.8 \\
\hline $\begin{array}{l}9-10 \hat{0}- \\
(n=96)\end{array}$ & 0.0 \\
\hline
\end{tabular}

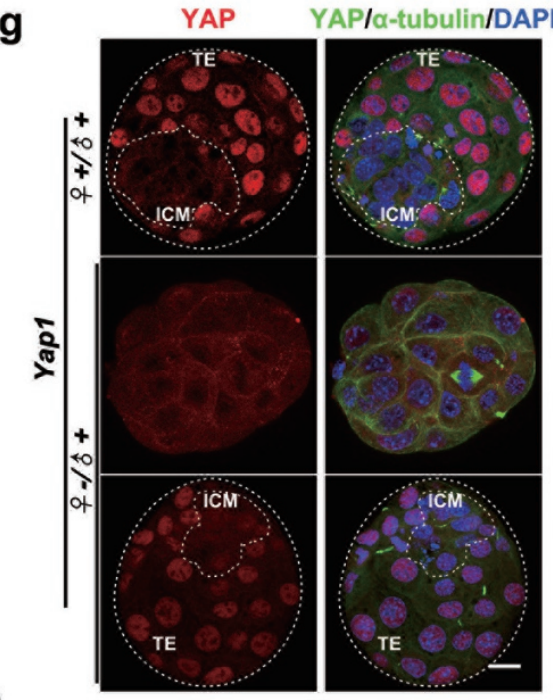

h

YAP YAP/a-tubulin/DAPI i

YAP

YAP/a-tubulin/DAPI j

YAP

YAP/a-tubulin/DAPI
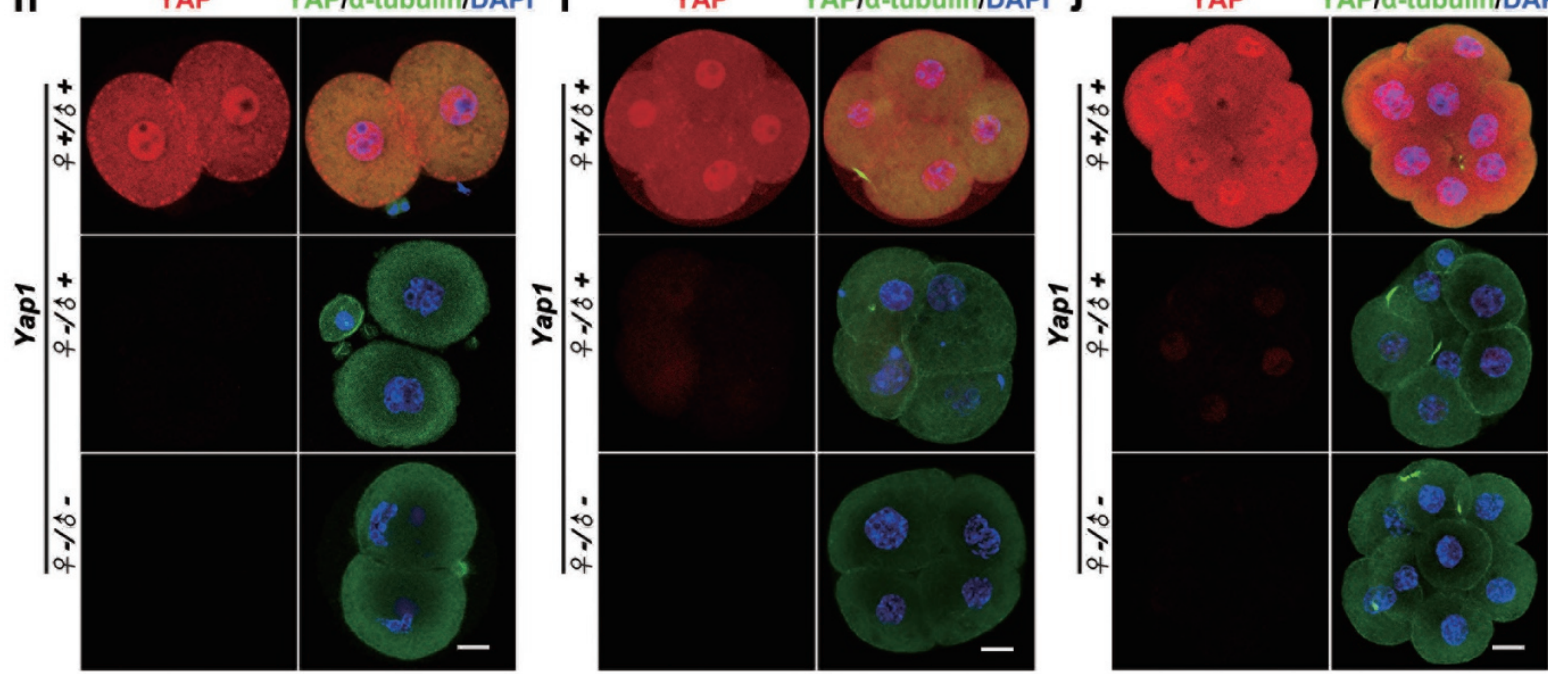

Cell Research | Vol 26 No 3 | March 2016 
3D) were not affected by maternal YAP deletion.

By embryonic day 3.5 (E3.5), normal blastocysts contain two types of cells: TE cells and ICM cells. Because most maternal YAP-deleted embryos failed to develop into blastocysts, we investigated whether the blastomeres in the developmentally arrested embryos acquired a TE cell fate or an ICM cell fate. Caudal type homeobox 2 (CDX2) is a functional marker for the TE, whereas the pluripotency-associated transcription factors Oct4 and Nanog are functional markers for the ICM. Few blasto-

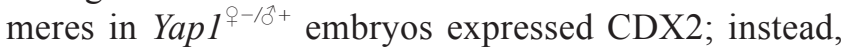
most blastomeres of Yap 1-deleted embryos expressed Nanog and OCT4 (Figure 3E and 3F). These results indicate that maternal YAP is required for proper differentiation of TE; in the absence of maternal YAP most blastomeres adopt an ICM cell fate.

\section{Identifying target genes of maternal YAP during zygotic genome activation}

To identify the YAP target genes in early embryos, we profiled the transcriptomes of GV oocytes and fourcell stage embryos derived from WT and Yap ${ }^{f / f}$; Gdf 9 Cre females (Figure 4A and Supplementary information, Figure S3A). Consistent with the inactive state of YAP in GV oocytes, transcriptomes of GV oocytes derived from WT and Yap $1^{f / f}$; Gdf9-Cre females were similar (Supplementary information, Figure S3B). There were only 186 transcripts downregulated and 87 transcripts upregulated in YAP-deleted oocytes. However, at the four-cell stage, the transcriptome of Yap $1^{\text {P-/ }}$ embryos was significantly different from that of WT embryos: 1292 transcripts were downregulated, and 2826 were upregulated in Yap ${ }^{\text {Q }} /^{3+}$ four-cell embryos (Figure 4B).

Gene ontology (GO) analyses revealed that many of the upregulated transcripts were associated with cell cycle (Supplementary information, Figure S3C). This is consistent with the prolonged two-cell stage and delayed embryogenesis upon Yap1 deletion. During MZT, many maternal transcripts are degraded in WT embryos to facilitate ZGA. Strikingly, $79.7 \%$ (2 254 in 2826 ) of the transcripts upregulated in Yap $1^{q-1 \widehat{\delta}^{+}}$embryos during the GV to four-cell transition were genes that would have been degraded in WT embryos (Supplementary information, Figure S3D). If only the top $10 \%$ abundant transcripts in oocytes are considered, $95.5 \%$ of the upregulated transcripts in $\mathrm{Yapl}^{+-1{ }^{2+}}$ embryos evaded degradation that is their normal fate (Figure 4C and Supplementary information, Figure S3D), indicating the importance of maternal YAP in MZT. Expression of representative transcripts is shown in Figure 4D and Supplementary information, Figure S3E.

On the other hand, the downregulated transcripts in Yap $^{\text {P-1/3+ }}$ embryos were significantly associated with protein translation (Figure 4E). Among these, 704 transcripts are upregulated during ZGA in WT embryos (from zygote stage to four-cell stage). These genes are potentially the YAP-TEAD-targeted early zygotic genes. When compared with published ChIP results of YAP in mouse embryonic stem cells [42], 23 genes were found to be YAP targets in early mouse embryos (Supplementary information, Table S1, FPKM (fragments per kilobase of transcript per million mapped reads) $>10$ in WT embryos at four-cell stage). The mRNA levels of selected genes in WT and Yap $1^{\text {q-13+ }}$ embryos determined by quantitative real-time PCR (qRT-PCR) (Figure 4F) were largely consistent with the RNA-seq data.

Among the 23 genes, Rpl13 (ribosomal protein L13), Rrm2 (ribonucleotide reductase M2), Alppl2 (alkaline phosphatase 2), Sfn (stratifin), Slc39a4 (solute carrier family 39, member 4) and Tead4 contain 1-3 putative TEAD-binding sites within $2000 \mathrm{bp}$ upstream of their transcription start sites [23]. All these genes were down-

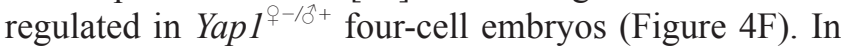
luciferase assay, promoters of Rpl13 and Rrm2 elicited strong responses to YAP-TEAD1 and YAP-TEAD4 (Figure 5A), but not to a YAP mutant that lacks affinity for TEADs (Figure 5B and 5C). Overexpression of YAPTEAD1 in GV oocytes also increased the expression of Rpl13 and Rrm2, as well as other putative YAP target genes (Bzw2, Klf2 and $\mathrm{Ncl}$; Figure 5D).

Figure 2 Maternal deletion of YAP causes female subfertility owing to impaired early embryogenesis. (A) Schematic diagram

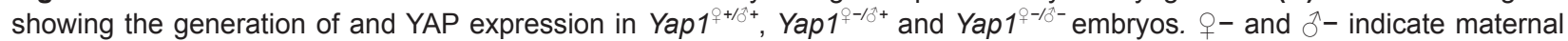
or paternal deletion of Yap1 allele. (B) Maternal YAP deletion causes a delay in early embryonic development. More than six mice were analyzed at each time point and numbers of embryos $(n)$ of two genotypes in the analysis are indicated. (C) Morphology of embryos collected from the oviducts or uteri of mice with indicated genotypes, at different time points after hCG administration and mating with adult WT males. Scale bar, $100 \mu \mathrm{m} . n>6$ for each genotype. (D) Maternal YAP deletion or maternal-paternal double YAP deletion causes defects in developing to blastocyst stage. Numbers of embryos $(n)$ analyzed are indicated. (E-F) Numbers of pups per litter (E) and cumulative numbers of pups per female $(\mathbf{F})(n \geq 7)$. Error bars indicate SEM. ${ }^{* *} P<0.001$, Student's $t$-test. (G) Immunofluorescent staining showing the expression of paternal Yap1 in Yap $1^{\circ-1 \delta^{*+}}$ em-

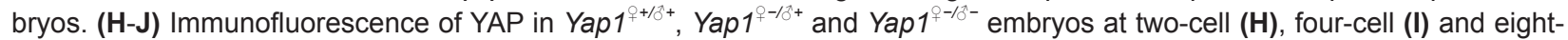
cell (J) stages. 


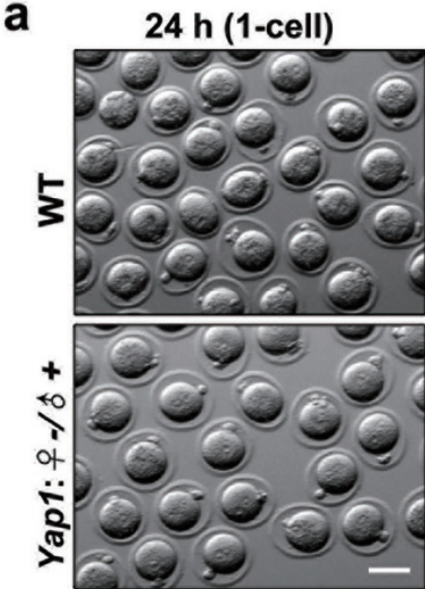

b

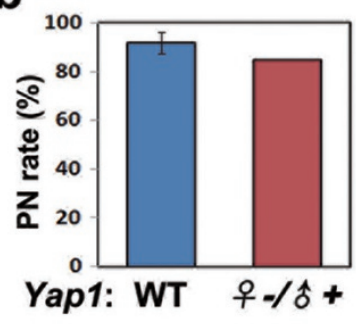

c

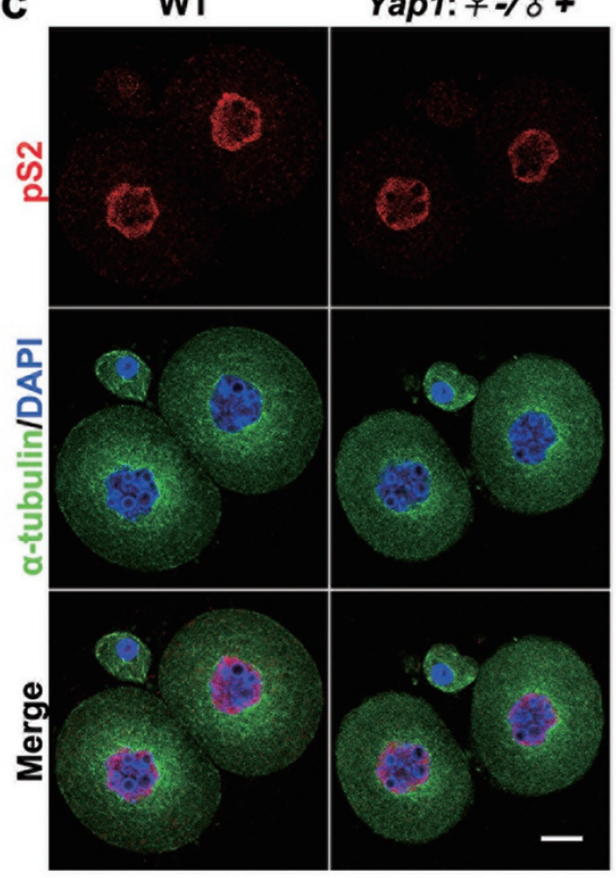

d

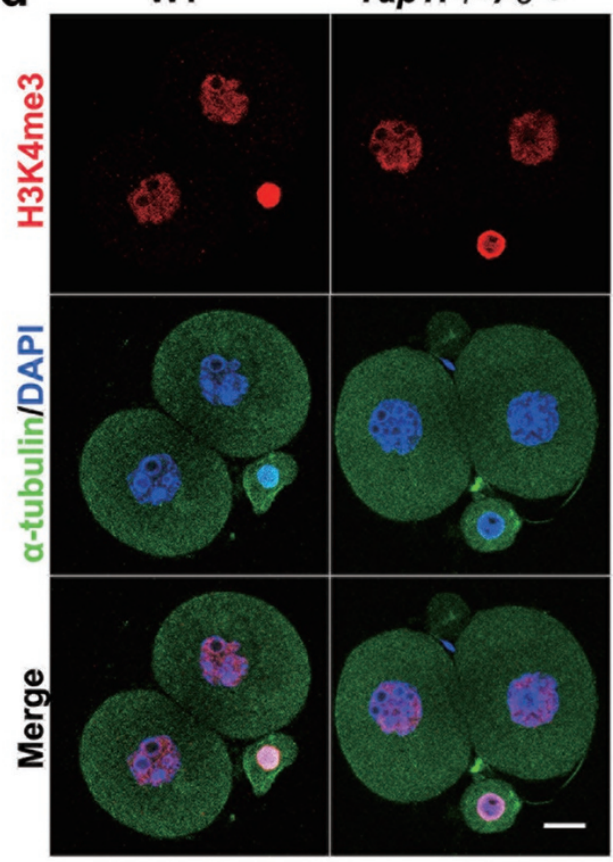

e
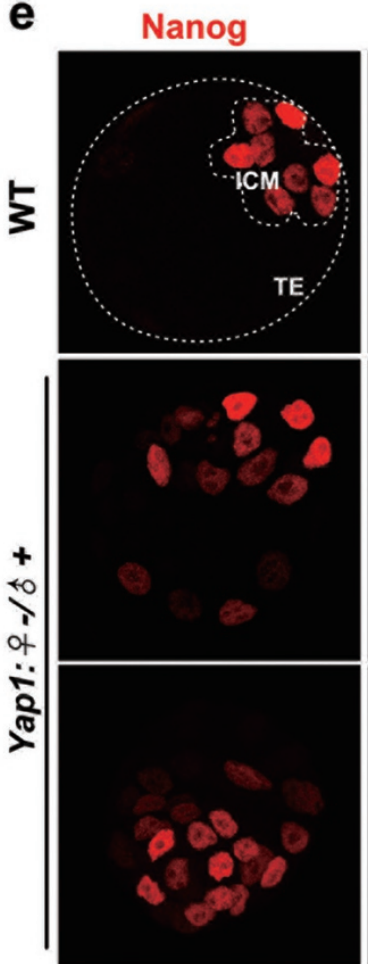

CDX2
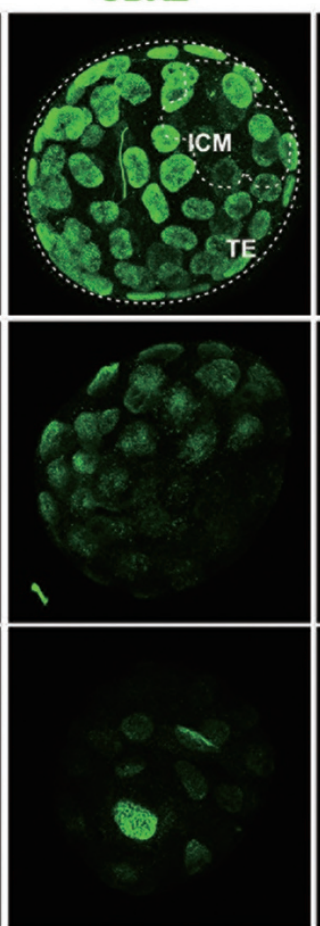

MERGE
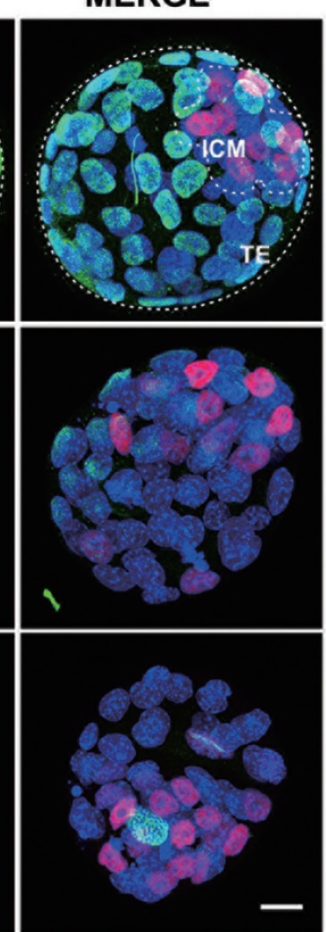

f

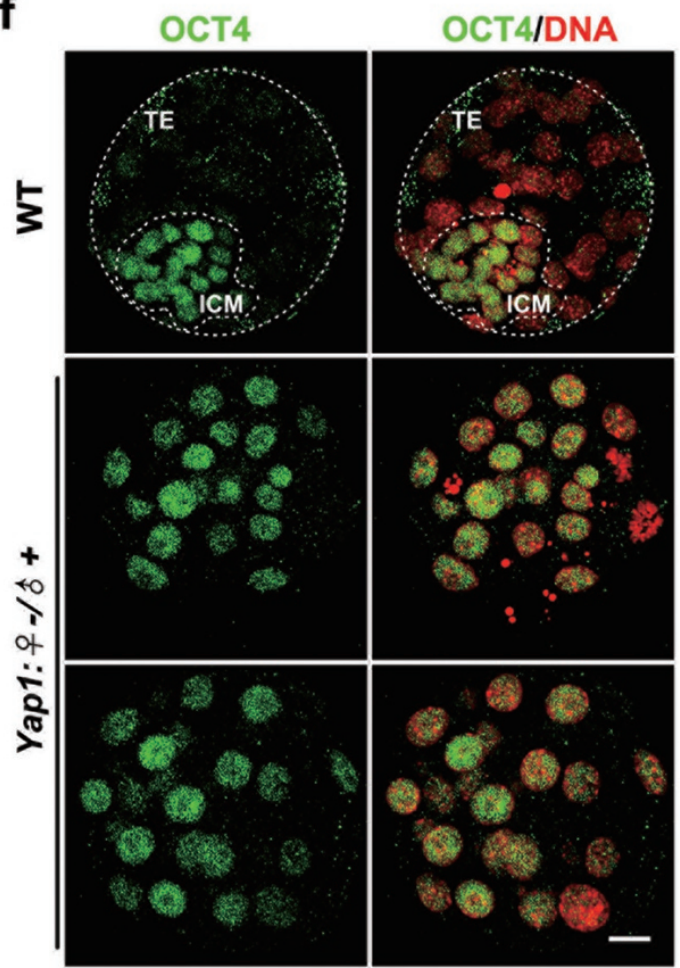

Figure 3 Embryogenesis defects in YAP-deleted embryos. (A) Morphology of zygotes derived from WT and Yap ${ }^{f / f t}$;Gdf9-Cre females after hCG injection and successful mating. Scale bar, $50 \mu \mathrm{m}$. (B) Pronucleus formation rates of WT and Yap1 ${ }^{\circ+1 /{ }^{+}}$ zygotes. Error bar indicates SEM. (C, D) Immunofluorescence showing RNA polymerase II phosphorylation (pS2) (C) and histone $\mathrm{H} 3$ trimethylation at lysine $4(\mathrm{H} 3 \mathrm{~K} 4 \mathrm{me} 3)(\mathrm{D})$ in WT and Yap $1^{\text {f-1/ }}$ embryos at the two-cell stage. Scale bar, $10 \mu \mathrm{m}$. (E) Immunofluorescence of Nanog and CDX2 in WT and Yap $1^{\text {- } / 3^{+}+}$embryos at the time when WT embryos develop to the blastocyst stage. TE, trophectoderm; ICM, inner cell mass. Scale bar, $10 \mu \mathrm{m}$. (F) Immunofluorescence of OCT4 in WT and $\mathrm{Yap}^{+1{ }^{+}+}$embryos at the time when WT embryos are at the blastocyst stage. Scale bar, $10 \mu \mathrm{m}$. 
Rpl13 and Rrm 2 are among the early expressed genes in human and mouse embryos (Supplementary information, Figure S4). Rpl13 encodes a ribosomal protein that promotes protein translation; $R r m 2$ encodes an essential component of ribonucleotide reductase that catalyzes the biosynthesis of deoxyribonucleotides [24]. The induction of these two genes by YAP-TEAD might improve the developmental competence of early embryos by increasing protein translation and DNA synthesis. Indeed, combined depletion of Rpl13 and Rrm 2 in WT zygotes reduced the developmental competence of embryos (Figure 5E-5G). The Rpl13- and Rrm2-depleted embryos were arrested at the morula stage before compaction (Figure 5F). This de-

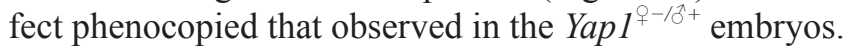
The expression of YAP target genes was compromised in Rpl13- and Rrm2-depleted embryos, as shown by qRTPCR results (Figure 5H). Taken together, maternal YAP is activated in early embryos and transcriptionally activate Rpll3 and Rrm2, which critically regulate developmental competence.

\section{LPA stimulates early embryogenesis by activating YAP}

In cultured cell lines, YAP activity and its subcellular distribution are controlled by the Hippo pathway [25]. LPA inhibits the Hippo pathway components LATS1/2, and therefore activates YAP [26]. LPA is present in many animal biological fluids including oviduct tubal fluid [27]. Significant nuclear localization of YAP was observed in four-cell embryos collected from the oviducts, but not in those cultured in vitro (Figure 6A and 6B). However, LPA treatment promoted YAP nuclear localization, as well as expression of downstream genes in cultured embryos (Figure 6B-6E). These results suggest that LPA activates YAP in early embryos in vivo.

M16 medium is widely used in oocyte culture but barely supports embryonic development (Figure 6F). Adding LPA to M16 medium significantly increased the development rates of WT embryos, but this effect was not observed in Yapl $^{\text {P-1 }} \widehat{\delta}^{\hat{+}}$ embryos (Figure $6 \mathrm{~F}$ and $6 \mathrm{G}$ ). In an embryo transfer experiment, foster mothers transferred with LPA-treated embryos gave birth to more pups than fosters that received control-treated embryos (Figure $6 \mathrm{H})$. These results suggest that LPA improves the developmental competence of preimplantation embryos by activating YAP and its downstream genes (Figure 7).

\section{Discussion}

The involvement of YAP-TEAD in mouse embryonic development has been reported [15, 16, 28, 29]. However, the contribution of maternal Yapl mRNA and protein was less clear before our study. Here we demonstrate a detrimental effect of maternal YAP deletion at two- to four-cell stage, and identify critical YAP-TEAD target genes in early embryos (Figure 7). Conventional Yap1 knockout results in developmental defects at E8.5 without affecting preimplantation development [29]. This suggests that maternal YAP stored in oocytes by app $^{+-}$ mother is sufficient to support development beyond the early stages. In addition, although YAP was reported to be important for survival, proliferation and differentiation in a wide range of somatic cell types, germ cell-specific Yapl knockout affected neither oogenesis nor spermatogenesis. Our observations highlight the developmental stage-specific role of maternal YAP in ZGA, and zygotic Yapl as an early zygotic gene required for embryogenesis when the maternal YAP is exhausted at the approximately four-cell stage.

The functions of YAP-TEAD and the upstream Hippo pathway have been reported in many cell types including embryonic stem cells; however, YAP target genes in specific physiological contexts are less understood. The most studied TEAD4 target gene in preimplantation embryos is $C d x 2$, which is crucial for TE specification [15, $30,31]$. But the defects in maternal YAP-depleted embryos occur as early as the two- to four-cell stage, at which time CDX2 is absent. This suggests that maternal YAP targets other early-expressed zygotic genes. By comparing the transcriptomes between WT and Yap $1^{\text {}-1 / \delta^{+}+}$mouse embryos, we have now identified novel YAP-TEAD-regulated early zygotic genes. Among them Rpll3 and Rrm 2 are direct target of YAP-TEADs. RPL13 and RRM2 may implement YAP's effect by promoting protein and DNA synthesis immediately after ZGA. Depletion of them in zygotes causes defects similar to maternal YAP deletion. Thus, expression of Rpll3 and Rrm 2 may be used as a new criterion in evaluating developmental competence of early mammalian embryos.

LPA has been reported to improve the developmental competence of cultured bovine embryos, but the underlying mechanism(s) was unclear [32-35]. Although LPA is known to activate YAP by inhibiting the Hippo pathway in cultured cell lines, a physiological context for this regulation has been lacking. Here we show YAP can mediate LPA's effect in improving the developmental competence of preimplantation embryos. LPA binds to albumin with high affinity and exists in most of commercial serum or albumin products $[26,36]$. Albumin is routinely added in human and mouse embryo culture media [37-39]. The protein-bound LPA, instead of albumin itself, may be the key bioactive molecule to support in vitro embryonic development. Considering that serum products are prone to contamination by unknown pathogens, LPA is a plausible and potentially safer substitute in making chemically defined embryo culture media. This justifies further investigation before LPA is to be adopted in human assisted 
a

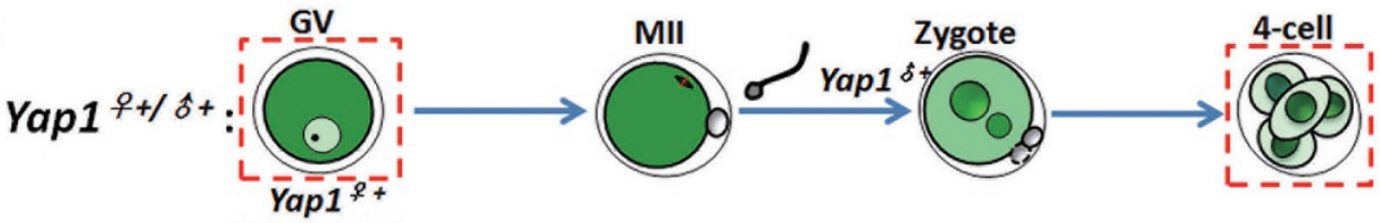

Yap1 우수의

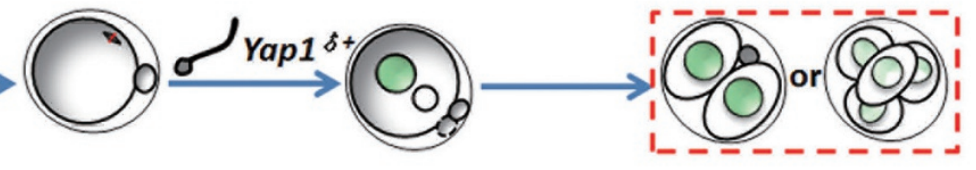

Samples used for RNA-seq

b

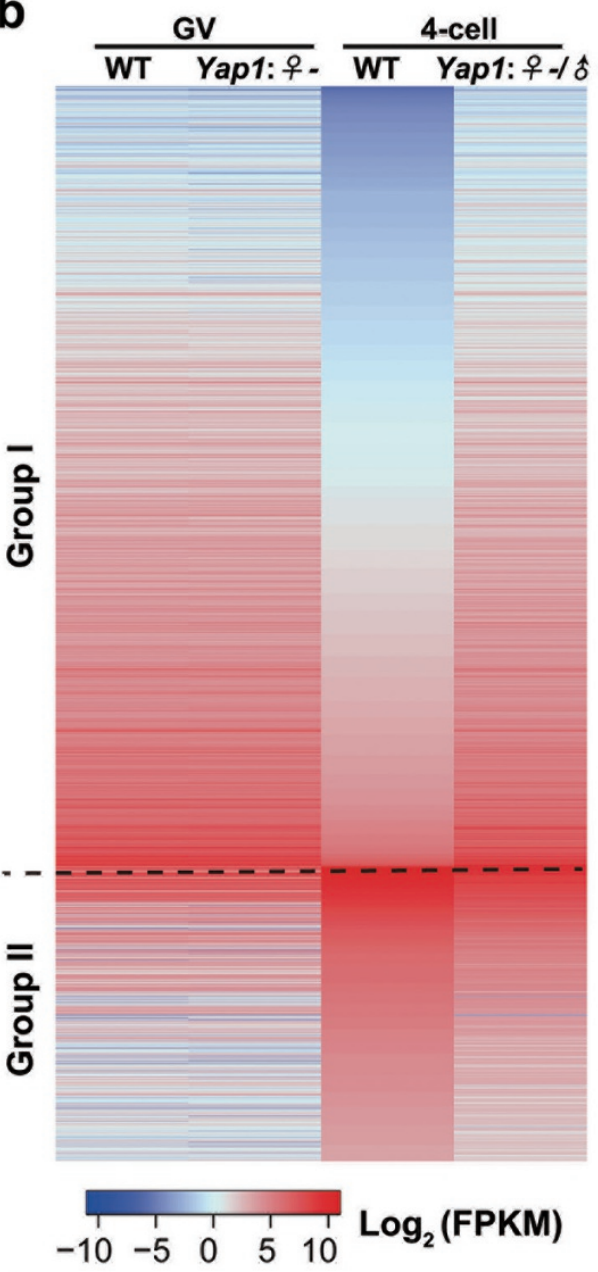

YAP concentration

C

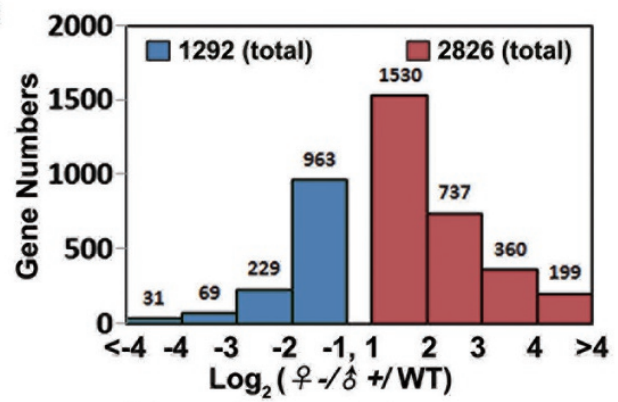

d

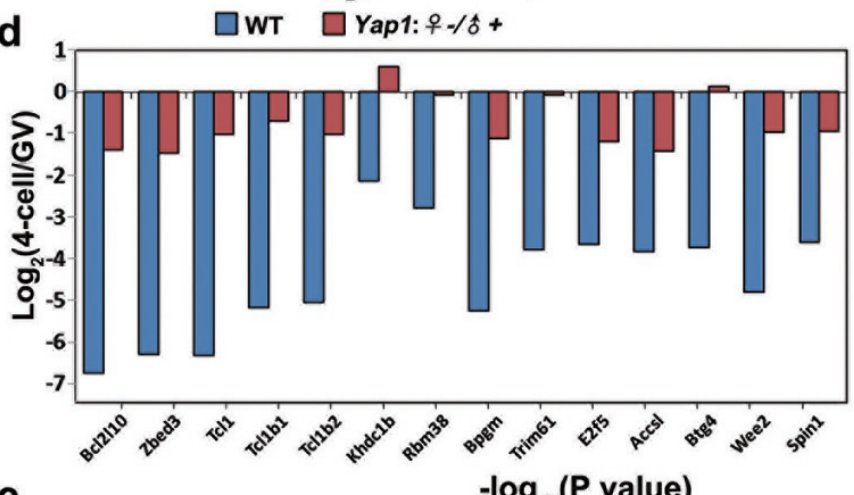

e

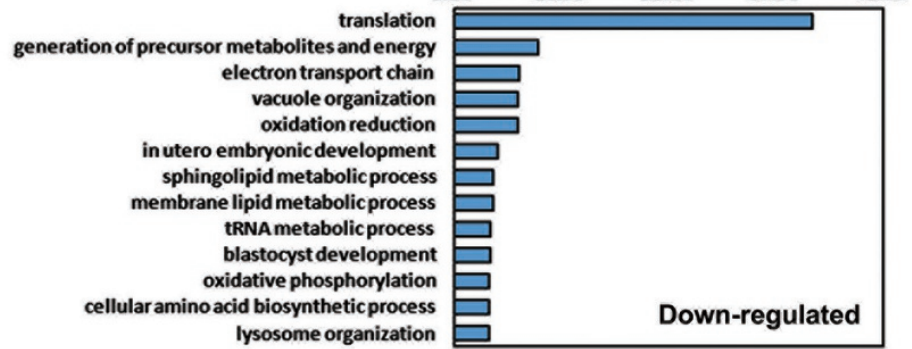

f

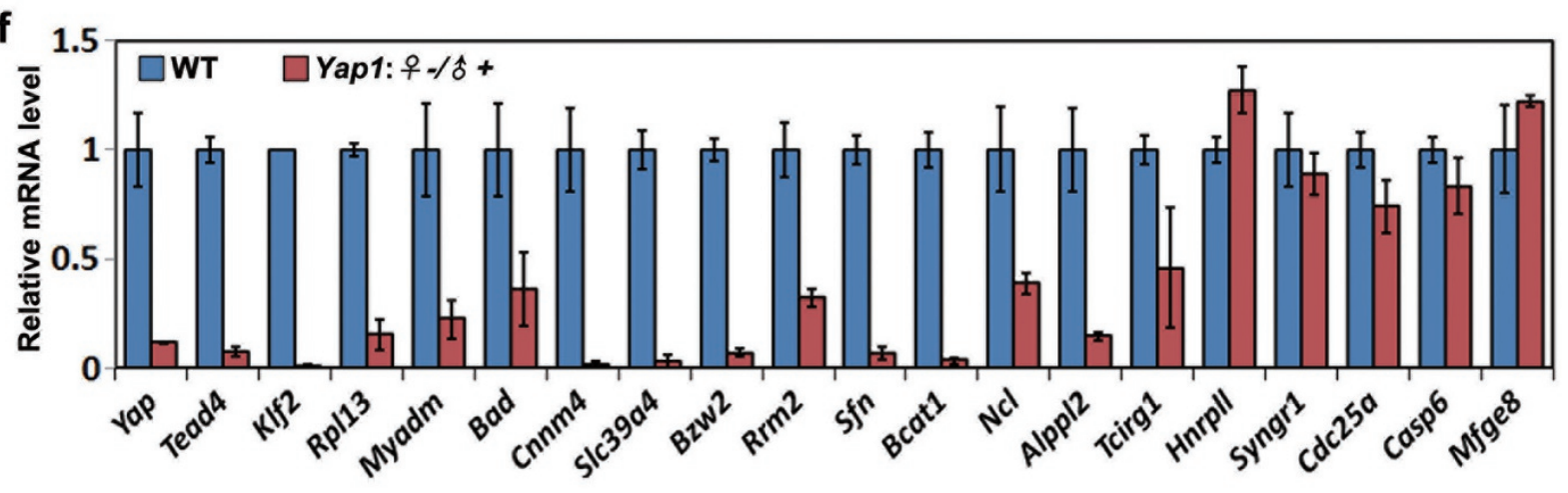


reproductive medicine.

\section{Materials and Methods}

Mice

Yap $1^{f / f l}$ mice [17] were a gift from Dr Duojia Pan and were crossed with Gdf9-Cre [40] or Ddx4-Cre [41] transgenic mice. Primers used for genotyping are listed in Supplementary information, Table S2. Animal care and experimental procedures were in accordance with the Animal Research Committee guidelines of Zhejiang University.

\section{Oocyte culture and microinjection}

Twenty-one-day-old mice were injected with pregnant mare's serum gonadotropin (PMSG) and were euthanized $44 \mathrm{~h}$ later. Oocytes at GV stage were cultured in mini-drops of M16 medium (M7292; Sigma-Aldrich) covered with mineral oil at $37{ }^{\circ} \mathrm{C}$ in $5 \%$ $\mathrm{CO}_{2}$. For microinjection, mRNAs were in vitro transcribed using SP6 message machine kit (Invitrogen, AM1450). siRNA was obtained from GenePharma and the sequences are shown in Supplementary information, Table S2. Microinjection of oocytes was performed using an Eppendorf microinjector.

Superovulation, fertilization and embryo culture

Pubertal female mice (21-23 days old) were intraperitoneally injected with 5 IU PMSG for $44 \mathrm{~h}$ and followed by 5 IU human chorionic gonadotropin (hCG). Sixteen $\mathrm{h}$ after the second injection, cumulus-oocyte complexes were collected from oviducts. To obtain early embryos, female mice were mated with adult WT males overnight after hCG administration. Zygotes and embryos were collected from oviducts or uteri at indicated time points posthCG injection, and cultured in KSOM (Sigma-Aldrich). For the experiments related to LPA treatments, zygotes were cultured in M16 with or without adding $10 \mu \mathrm{M}$ LPA. Embryo morphology was observed and images were acquired using a Nikon SMZ1500 stereoscope.

\section{Embryo transfer}

Zygotes were collected as described above and were cultured in M16 with or without adding $10 \mu \mathrm{M}$ LPA for $80 \mathrm{~h}$. Next, the embryos were transferred into the uteri of pseudopregnant ICR female mice ( 15 embryos per mouse). The litter sizes were determined at birth.

$R N A$ extraction and $q R T-P C R$

Total RNA was extracted using RNeasy Mini kit (Qiagen) according to the manufacturer's instructions. qRT-PCR analyses were performed using a Power SYBR Green PCR Master Mix (Life technologies) on an Applied Biosystems 7500 PCR System. Relative mRNA levels were calculated by normalizing to the levels of endogenous Gapdh mRNA (internal control) using Microsoft EXCEL. For each experiment, qPCR reactions were done in triplicate. Primer sequences are listed in Supplementary information, Table S2.

\section{RNA-seq and library construction}

GV oocytes and four-cell embryos were collected from WT and Yap ${ }^{f / f}$; Gdf9-Cre mice (100 oocytes or embryos per sample). Total RNA was extracted from the samples using the RNeasy Plus Micro kit (Qiagen) following the manufacturer's protocol. Before RNA isolation, $2 \times 10^{6}$ mRNA-RFP was added to calculate mRNA copy number in each sample. Extracted total RNA was used to build a sequencing library using the NEB Next Ultra RNA Library Prep Kit for Illumina. We sequenced the library by Illumina Hiseq 2500 and aligned RNA-seq reads to Mus musculus UCSC mm9 references with the Tophat software (http://tophat.cbcb.umd.edu/), and calculated the FPKM of each gene using Cufflinks (http://cufflinks.cbcb.umd.edu). The amount of total mRNA was calculated based on the FPKM of exogenous RFP. GO analysis for enrichment of genes was determined using the Database for Annotation, Visualization and Integrated Discovery. The RNA-seq data have been deposited in Gene Expression Omnibus under the accession number GSE74344.

\section{Confocal microscopy}

Oocytes and embryos were fixed for $30 \mathrm{~min}$ in $4 \%$ paraformaldehyde dissolved in PBS. After blocking in 1\% BSA in PBS, oocytes were incubated with primary antibodies diluted in the blocking solution for $1 \mathrm{~h}$. After three washes, oocytes were incubated with secondary antibodies for $30 \mathrm{~min}$, and then counterstained with $5 \mu \mathrm{g} / \mathrm{ml}$ 4',6-diamidino-2-phenylindole or propidium iodide for $10 \mathrm{~min}$. Oocytes were mounted and examined with a confocal laser scanning microscope (Zeiss LSM 710, Carl Zeiss AG, Germany). The primary antibodies used are listed in Supplementary information, Table S3.

\section{Western blot analysis}

Total protein extract from 100 oocytes was separated by SDSPAGE and transferred to PVDF membranes (Millipore). After incubation with primary antibodies and an HRP-linked secondary antibody, bands on the membranes were detected using an Enhanced Chemiluminescence Detection Kit (Amersham). Antibod-

Figure 4 Maternal Yap1-knockout zygotes are defective in maternal-zygotic transition (MZT). (A) Schematic diagram showing the samples used for RNA-seq. (B) Heatmap illustration showing differentially expressed transcripts in WT and Yap1-deleted GV oocytes and four-cell stage embryos. Group I, transcripts that are degraded (fold change > 2) during GV to four-cell transition in WT embryos but remain stable in Yap1-deleted four-cell embryos. Group II, transcripts that are induced (fold change $>2$ and FPKM > 10 in WT four-cell embryos) from GV stage to four-cell stage in WT embryos but are significantly less upregulated in Yap1-deleted four-cell embryos. (C) Numbers of transcripts that are repressed (blue) or abnormally upregulated (red) in Yap $1^{+/ /^{+}+}$four-cell embryos when compared with WT four-cell embryos. (D) RNA-seq results of selected transcripts that are downregulated after fertilization in WT oocytes (blue) but remain stable in Yap $1^{\text {to }}$ to $^{\text {t }}$ four-cell embryos (red). (E) Gene ontolo-

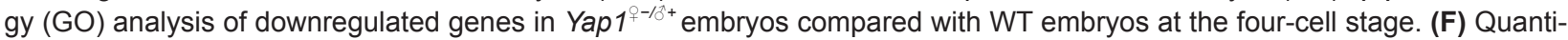
tative real-time PCR (qRT-PCR) results showing the expression levels of indicated transcripts in WT and Yap1 ${ }^{1^{-1 / 3}+}$ embryos at the four-cell stage. Error bars indicate SEM. 
a

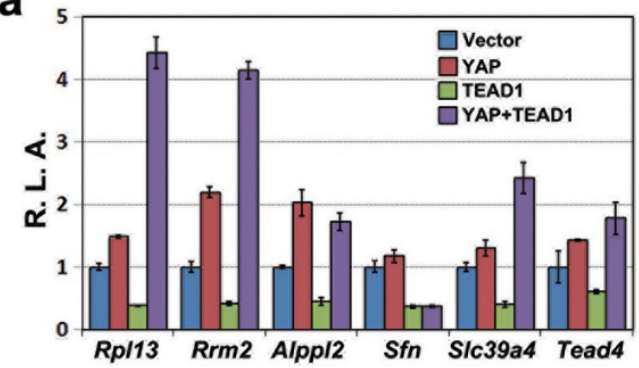

d
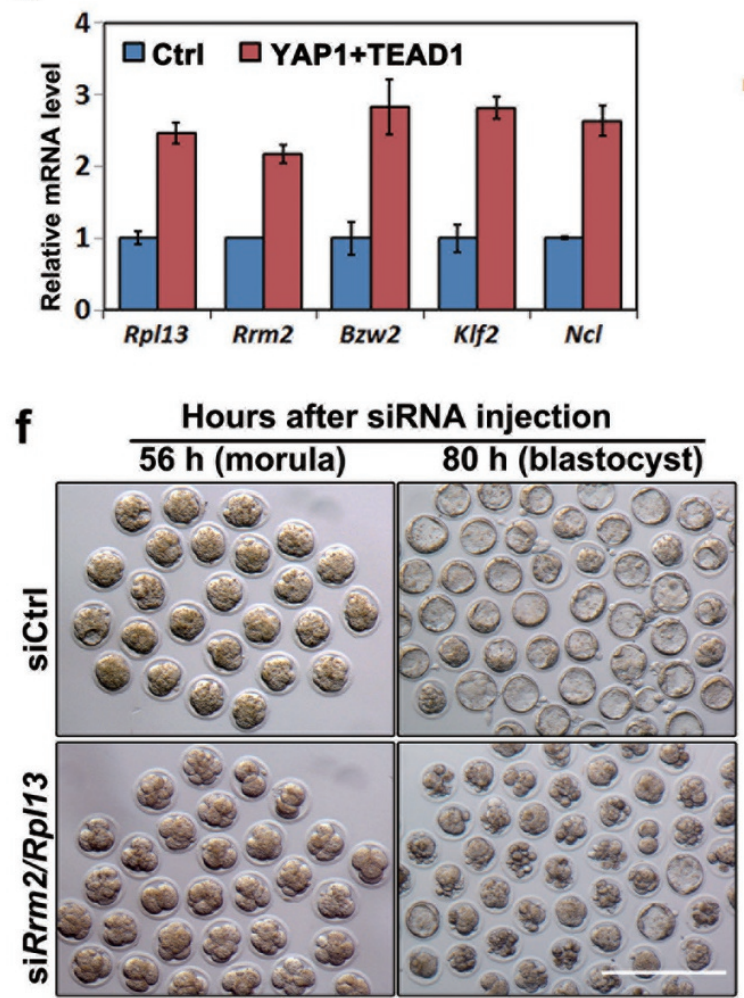

e b
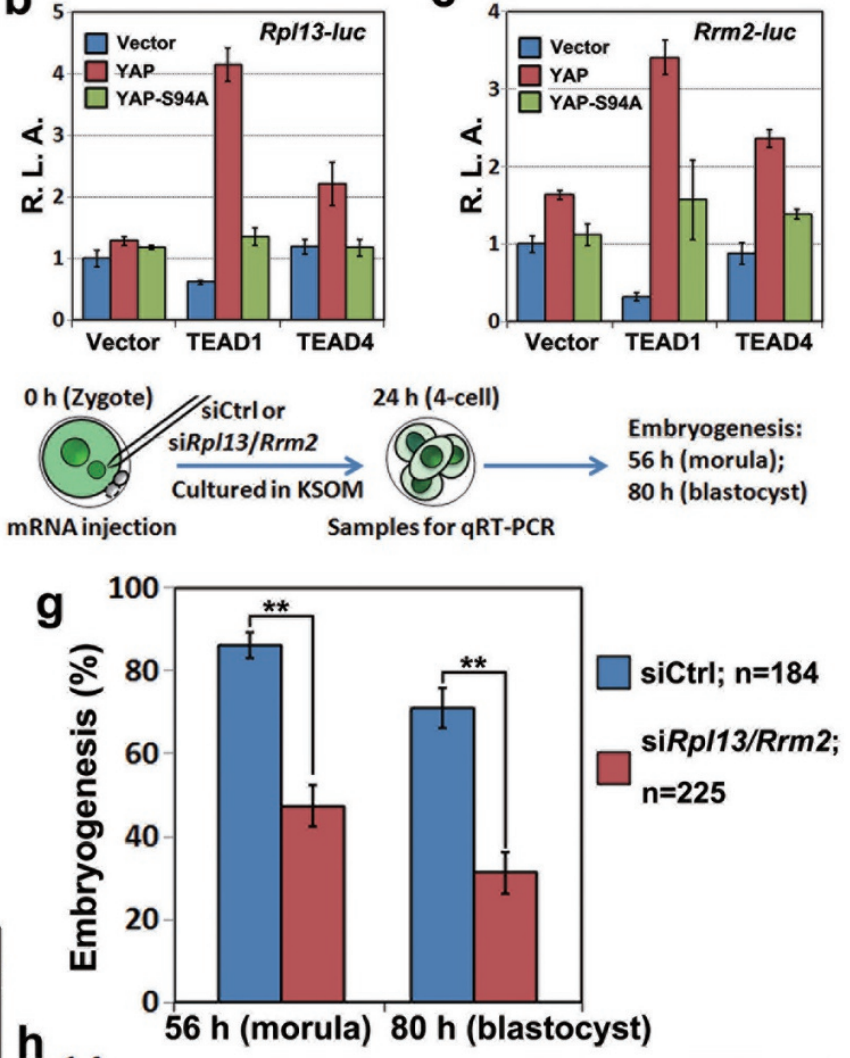

h

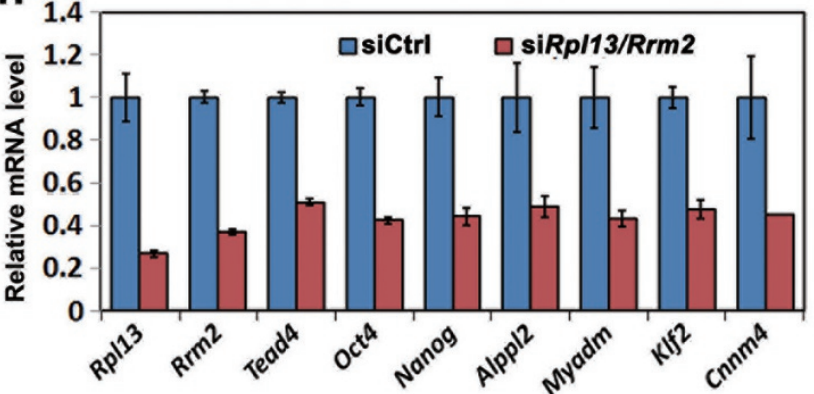

Figure 5 Rp/13 and Rrm2 are key YAP target genes that render developmental competence to preimplantation embryos. (A) Luciferase reporter activity of promoters of indicated genes in response to YAP-TEADs. RLA, relative luciferase activity. Error bars indicate SEM. (B-C) Luciferase reporter activity of Rp/13 (B) and Rrm2 (C) promoters in response to YAP-TEADs. Error bars indicate SEM. (D) Overexpression of YAP-TEAD1 in oocytes induces the expression of indicated genes. Error bars indicate SEM. (E) Schematic illustration of RNAi in zygotes. (F) Morphology of zygotes injected with control siRNA or siRNAs targeting $R p / 13$ and $R r m 2$ at $56 \mathrm{~h}$ and $80 \mathrm{~h}$ after injection. Scale bar, $200 \mu \mathrm{m}$. (G) Quantification of embryos that develop to the morula stage $(56 \mathrm{~h})$ and blastocyst stage $(80 \mathrm{~h})$ after injection with control siRNA or siRNAs targeting Rp/13 and Rrm2. Numbers of observed embryos are indicated. Error bars indicate SEM. ${ }^{* *} P<0.01$, Student's $t$-test. (H) qRT-PCR results showing expression of YAP target genes in four-cell embryos after Rp/13/Rrm2 depletion in zygotes. Error bars indicate SEM.

ies and the dilutions used are indicated in Supplementary information, Table S3.

\section{Histological analysis}

Formalin-fixed, paraffin-embedded samples were sectioned at $5-\mu \mathrm{m}$ thickness and stained with hematoxylin and eosin. For immunofluorescent staining, sections were sequentially probed with primary antibodies and conjugated secondary antibodies (Molecular Probes). Digital images were acquired using an epifluorescent microscope (Nikon Eclipse 80i). Antibodies used are listed in Supplementary information, Table S3.

\section{Cell culture, plasmid transfection and luciferase assay}

HEK293 cells were grown in DMEM (Invitrogen) supplemented with $10 \%$ fetal bovine serum. Transient plasmid transfections were done using Lipofectamine 2000 (Invitrogen). For luciferase assay, cells were transfected with luciferase reporters with or without plasmids encoding components of YAP-TEAD complex. 
a
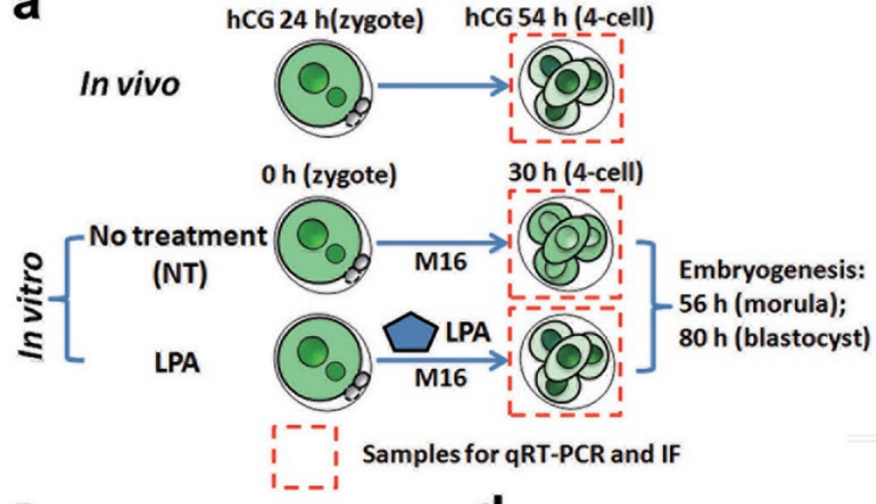

C
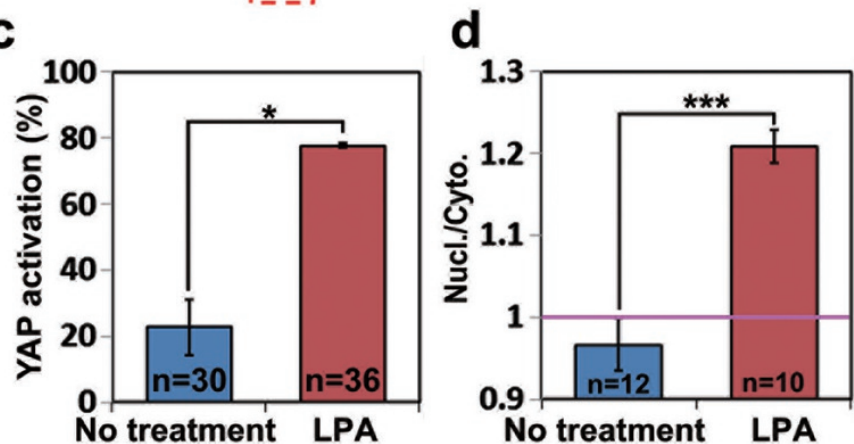

$\mathbf{f}$

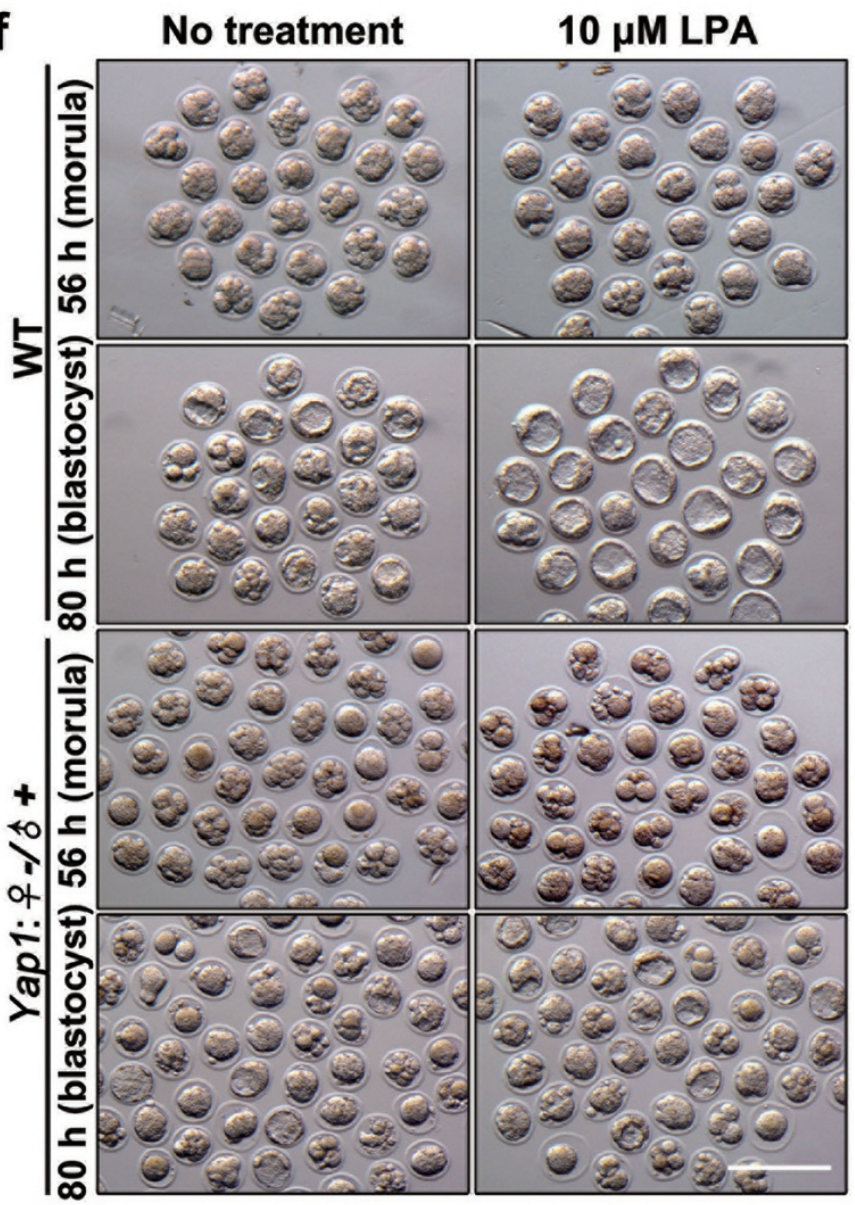

b Culture from zygote (in vitro)

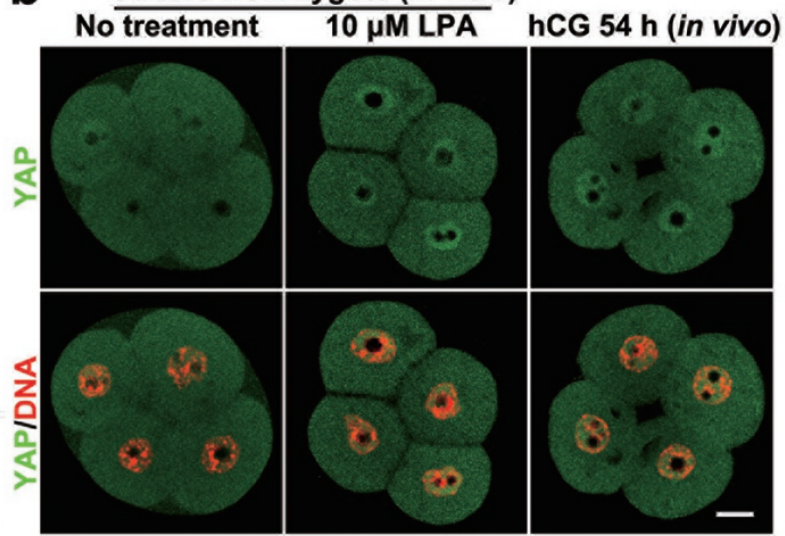

e

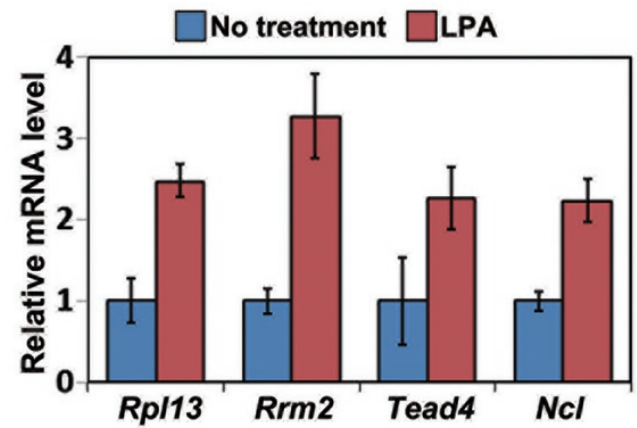

g

$\square$ WT; NT (n=257) $\square$ Yap1: 우-/ \& + ; NT (n=204)

$\square$ WT; LPA $(n=240) \square$ Yap1: 우 $-/ \delta$ + ; LPA $(n=190)$

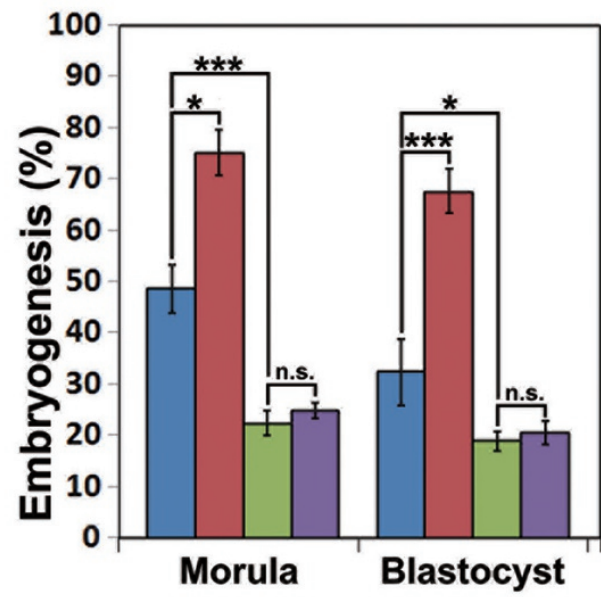

h

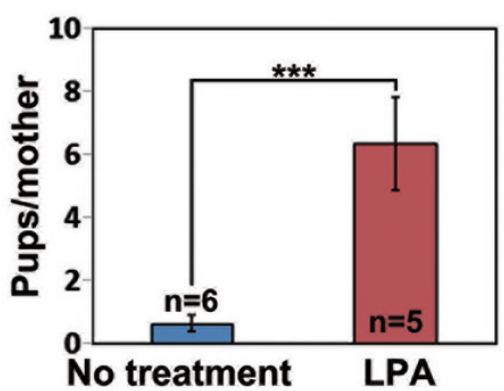


Expression of Renilla luciferase was served as loading control. After 48 h, cells were lysed in Passive Lysis Buffer (Promega) and subjected to luciferase assay following the manufacturer's instructions (Dual luciferase system, Promega).

\section{Statistical analysis}

Results are given as means \pm SEM; each experiment included at least three independent samples and was repeated at least three times. Group comparisons were made by two-tailed unpaired Student's $t$-tests. ${ }^{*} P<0.05 ;{ }^{* *} P<0.01$, and ${ }^{* * *} P<0.001$.

\section{Acknowledgments}

We thank Dr Duojia Pan for Yapl flox mice. This study was funded by the National Basic Research Program of China (2012CB944403) and the National Natural Science Foundation of China (81172473, 91519313 and 31371449).

\section{Author Contributions}

CY, SYJ, QQS, JJZ, YFY and LYY conducted experiments. YD and FT conducted informatics analyses of RNA sequencing results. JQ, FT and HYF conceived and designed the experiments and wrote the paper.

\section{Competing Financial Interests}

The authors declare no conflict of interest.

\section{References}

1 Sun F, Fang H, Li R, et al. Nuclear reprogramming: the zygotic transcription program is established through an "eraseand-rebuild" strategy. Cell Res 2007; 17:117-134.

2 Li L, Lu X, Dean J. The maternal to zygotic transition in mammals. Mol Aspects Med 2013; 34:919-938.

3 Schier AF. The maternal-zygotic transition: death and birth of RNAs. Science 2007; 316:406-407.

4 Schultz RM. The molecular foundations of the maternal to zygotic transition in the preimplantation embryo. Hum Reprod Update 2002; 8:323-331.

5 Tadros W, Lipshitz HD. The maternal-to-zygotic transition: a play in two acts. Development 2009; 136:3033-3042.

6 Walser CB, Lipshitz HD. Transcript clearance during the maternal-to-zygotic transition. Curr Opin Genet Dev 2011; 21:431-443.

7 Fulka J, Jr, Mrazek M, Fulka H, Loi P. Mammalian oocyte therapies. Cloning Stem Cells 2005; 7:183-188.

8 Schmiady H, Kentenich H, Stauber M. [Developmental arrest of fertilized human oocytes in the pronuclear stage after in vitro fertilization (IVF)]. Geburtshilfe Frauenheilkd 1987; 47:406-409.

9 Menezo YJ, Sakkas D, Janny L. Co-culture of the early human embryo: factors affecting human blastocyst formation in vitro. Microsc Res Tech 1995; 32:50-56.

10 Liang HL, Nien CY, Liu HY, Metzstein MM, Kirov N, Rushlow C. The zinc-finger protein Zelda is a key activator of the early zygotic genome in Drosophila. Nature 2008; 456:400403.

11 Yan L, Yang M, Guo H, et al. Single-cell RNA-Seq profiling of human preimplantation embryos and embryonic stem cells. Nat Struct Mol Biol 2013; 20:1131-1139.

12 Gallardo TD, John GB, Shirley L, et al. Genomewide discovery and classification of candidate ovarian fertility genes in the mouse. Genetics 2007; 177:179-194.

13 Zhao B, Ye X, Yu JD, et al. TEAD mediates YAP-dependent gene induction and growth control. Gene Dev 2008; 22:19621971.

14 Chen LM, Chan SW, Zhang XQ, et al. Structural basis of YAP recognition by TEAD4 in the Hippo pathway. Gene Dev 2010; 24:290-300.

15 Yagi R, Kohn MJ, Karavanova I, et al. Transcription factor TEAD4 specifies the trophectoderm lineage at the beginning of mammalian development. Development 2007; 134:38273836.

16 Nishioka N, Inoue K, Adachi K, et al. The Hippo signaling pathway components Lats and Yap pattern Tead4 activity to distinguish mouse trophectoderm from inner cell mass. Dev Cell 2009; 16:398-410.

17 Zhang N, Bai H, David KK, et al. The Merlin/NF2 tumor suppressor functions through the YAP oncoprotein to regulate tissue homeostasis in mammals. Dev Cell 2010; 19:27-38.

18 Lan ZJ, Xu X, Cooney AJ. Differential oocyte-specific expression of Cre recombinase activity in GDF-9-iCre, Zp3cre, and Msx2Cre transgenic mice. Biol Reprod 2004; 71:14691474.

19 Varelas X. The Hippo pathway effectors TAZ and YAP in development, homeostasis and disease. Development 2014; 141:1614-1626.

20 Huang J, Wu S, Barrera J, Matthews K, Pan D. The Hippo signaling pathway coordinately regulates cell proliferation and apoptosis by inactivating Yorkie, the Drosophila homolog of YAP. Cell 2005; 122:421-434.

Figure 6 YAP mediates the lysophosphatidic acid (LPA) signaling to improve developmental competence of preimplantation embryos. (A) Schematic diagram showing LPA treatment of early embryos in vitro. (B) Localization of YAP in four-cell embryos developed in vivo and in vitro (with or without LPA treatment). Scale bar, $10 \mu \mathrm{m}$. (C) Percentage of blastomeres with significant localization of YAP in the nucleus in four-cell stage embryos cultured with or without LPA. Error bars indicate SEM. Numbers of observed embryos are indicated. ${ }^{*} P<0.05$, Student's $t$-test. (D) Ratios of YAP immunofluorescence signals in the nucleus (Nucl.) vs in the cytoplasm (Cyto.) with or without LPA treatment. Error bars indicate SEM. ${ }^{* * *} P<0.001$, Student's $t$-test. (E) LPA treatment activates the expression of YAP-TEAD target genes in the 4-cell stage embryos. Error bars indicate SEM. (F-G) Morphology (F) and quantification (G) showing that LPA treatment improves the developmental competence of WT embryos, but not Yap $1^{\not-1 /+}$ embryos. Numbers of embryos observed are indicated. Error bars indicate SEM. ${ }^{*} P<$ $0.05,{ }^{* * *} P<0.001$, Student's $t$-test. NS, not significant; NT, no treatment. Scale bar, $200 \mu \mathrm{m}$. (H) Embryo transfer experiments revealing that more embryos develop to term after LPA treatment. Numbers of foster mothers used are indicated. Error bars indicate SEM. ${ }^{* \star *} P<0.001$, Student's $t$-test. 


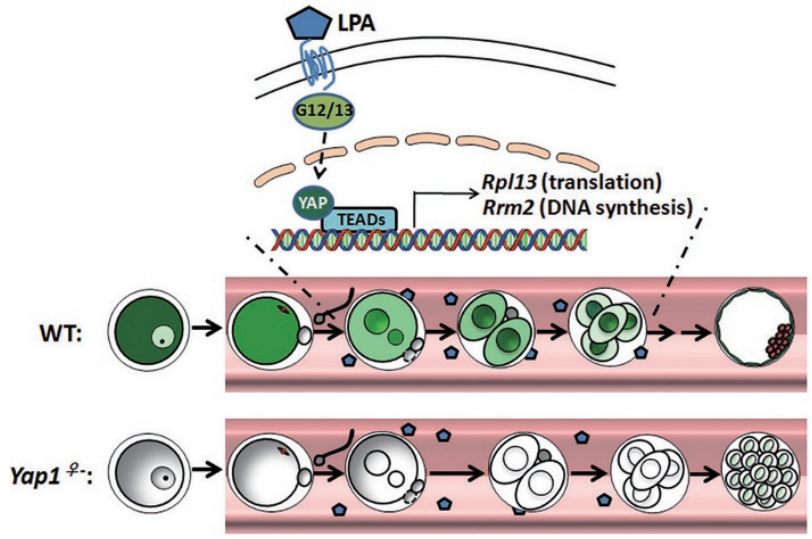

Figure 7 Schematic diagram showing functions of maternal YAP in early embryonic development. During early development of mammalian embryos, LPA in the tubal fluid binds to its G-protein coupled receptor at the plasma membrane, and induces the activation of YAP by inhibiting LATS1/2. YAP then enters the nucleus and binds with TEADs. Together, they induce the expression of Rp/13 and Rrm2, the two key YAP-TEAD target genes in early mouse embryos. The expression of $R p / 13$ and $R r m 2$ facilitates protein translation and DNA synthesis, which in turn increases the expression of genes associated with MZT and ultimately promotes early embryonic development. In maternal Yap1-knockout embryos, LPA fails to activate Rp/13 and Rrm2 expression, resulting in a decrease in developmental competence and embryogenesis failure before compaction.

21 Shen S, Guo X, Yan H, et al. A miR-130a-YAP positive feedback loop promotes organ size and tumorigenesis. Cell Res 2015; 25:997-1012.

22 Zhang W, Gao Y, Li P, et al. VGLL4 functions as a new tumor suppressor in lung cancer by negatively regulating the YAPTEAD transcriptional complex. Cell Res 2014; 24:331-343.

23 Anbanandam A, Albarado DC, Nguyen CT, Halder G, Gao X, Veeraraghavan S. Insights into transcription enhancer factor 1 (TEF-1) activity from the solution structure of the TEA domain. Proc Natl Acad Sci USA 2006; 103:17225-17230.

24 Heidel JD, Liu JY, Yen Y, et al. Potent siRNA inhibitors of ribonucleotide reductase subunit RRM2 reduce cell proliferation in vitro and in vivo. Clin Cancer Res 2007; 13:22072215.

25 Zhao B, Wei X, Li W, et al. Inactivation of YAP oncoprotein by the Hippo pathway is involved in cell contact inhibition and tissue growth control. Genes Dev 2007; 21:2747-2761.

26 Yu FX, Zhao B, Panupinthu N, et al. Regulation of the Hippo-YAP pathway by G-protein-coupled receptor signaling. Cell 2012; 150:780-791.

27 Sheng X, Yung YC, Chen A, Chun J. Lysophosphatidic acid signalling in development. Development 2015; 142:13901395.

28 Lorthongpanich C, Messerschmidt DM, Chan SW, Hong W, Knowles BB, Solter D. Temporal reduction of LATS kinases in the early preimplantation embryo prevents ICM lineage differentiation. Genes Dev 2013; 27:1441-1446.
29 Morin-Kensicki EM, Boone BN, Howell M, et al. Defects in yolk sac vasculogenesis, chorioallantoic fusion, and embryonic axis elongation in mice with targeted disruption of Yap65. Mol Cell Biol 2006; 26:77-87.

30 Strumpf D, Mao CA, Yamanaka Y, et al. Cdx2 is required for correct cell fate specification and differentiation of trophectoderm in the mouse blastocyst. Development 2005; 132:20932102.

31 Chen L, Yabuuchi A, Eminli S, et al. Cross-regulation of the Nanog and Cdx2 promoters. Cell Res 2009; 19:1052-1061.

32 Zhang JY, Jiang Y, Lin T, Kang JW, Lee JE, Jin DI. Lysophosphatidic acid improves porcine oocyte maturation and embryo development in vitro. Mol Reprod Dev 2015; 82:66-77.

33 Torres AC, Boruszewska D, Batista M, et al. Lysophosphatidic acid signaling in late cleavage and blastocyst stage bovine embryos. Mediators Inflamm 2014; 2014:678968.

34 Kobayashi T, Yamano S, Murayama S, Ishikawa H, Tokumura A, Aono T. Effect of lysophosphatidic acid on the preimplantation development of mouse embryos. Febs Lett 1994; 351:38-40.

35 Jo JW, Jee BC, Suh CS, Kim SH. Addition of lysophosphatidic acid to mouse oocyte maturation media can enhance fertilization and developmental competence. Hum Reprod 2014; 29:234-241.

36 Iglesias J, Abernethy VE, Wang Z, Lieberthal W, Koh JS, Levine JS. Albumin is a major serum survival factor for renal tubular cells and macrophages through scavenging of ROS. Am J Physiol 1999; 277:F711-F722.

37 Dandekar PV, Glass RH. Development of two-cell mouse embryos in protein-free and protein-supplemented media. $J$ In Vitro Fert Embryo Transf 1990; 7:107-113.

38 Caro CM, Trounson A. The effect of protein on preimplantation mouse embryo development in vitro. J In Vitro Fert Embryo Transf 1984; 1:183-187.

39 Andersen CY, Hay-Schmidt A, Byskov AG, Hornnes P, Starup J. [Human serum albumin as the protein source in culture of human oocytes, spermatozoa and pre-embryos]. Ugeskr Laeger 1991; 153:3393-3395.

40 Lan ZJ, Xu X, Cooney AJ. Differential oocyte-specific expression of Cre recombinase activity in GDF-9-iCre, Zp3cre, and Msx2Cre transgenic mice. Biol Reprod 2004; 71:14691474.

41 Gallardo T, Shirley L, John GB, Castrillon DH. Generation of a germ cell-specific mouse transgenic Cre line, Vasa-Cre. Genesis 2007; 45:413-417.

42 Lian I, Kim J, Okazawa H, et al. The role of YAP transcription coactivator in regulating stem cell self-renewal and differentiation. Genes Dev 2010; 24:1106-1118.

(Supplementary information is linked to the online version of the paper on the Cell Research website.)

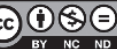
This work is licensed under a Creative Commons Attribution-NonCommercial-NoDerivs 4.0 Unported License. The images or other third party material in this article are included in the article's Creative Commons license, unless indicated otherwise in the credit line; if thematerial is not included under the Creative Commons license, users will need to obtain permission from the license holder to reproduce the material. To view a copy of this license, visit http://creativecommons.org/licenses/by-nc-nd/4.0/ 